\title{
Peak expiratory flow rate and Pulse Pressure values during the Luteal and Menstruation Phases of the Menstrual Cycle
}

\author{
*11OMOROGIUWA, A; OJIEMUDIA, M \\ Department of Physiology, School of Basic Medical Sciences, College of Medical Sciences, University of Benin, Benin city \\ Corresponding author: Dr Omorogiuwa A \\ E-mail: ask4ade2006@yahoo.com Phone number: +234(0)7039460340
}

\begin{abstract}
This study assessed the peak expiratory flow rate and pulse pressure during the luteal and menstruation phases of the menstrual cycle. The peak expiratory flow rate and pulse pressure were measured using the Wright's Peak Flow Meter and Mercury Sphygmomanometer respectively. The peak expiratory flow rate and pulse pressure in the luteal phase were $420.80 \pm 12.27$ and $42.72 \pm 2.52$ respectively. While the peak expiratory flow rate and pulse pressure in the menstruation phase were $378.40 \pm 11.85$ and $35.36 \pm 1.95$ respectively. The peak expiratory flow rate and the pulse pressure in the luteal phase were significantly higher $(\mathrm{p}<0.05)$ than the values during menstruation. The finding may be as a result of the relative increase in progesterone and estrogen levels in the luteal phase. The study thus shows an improved respiratory function and vasodilation during the luteal phase of the menstrual cycle (C) JASEM

http://dx.doi.org/10.4314/jasem.v19i4.8
\end{abstract}

\section{KEYWORDS: Menstruation, Luteal, Menstrual cycle ,Pulse Presuure, PEFR}

\section{Introduction}

The physiologic interplay of hormones of the menstrual cycle distinctly divides it into the estrogen dependent proliferative (follicular) phase and the progesterone dependent secretory (luteal) phase. The menstrual cycle ranges from 21-35 days. For the purpose of clarity if we take a menstrual cycle of 28 days the proliferative phase is equal to the secretory phase and just mid-way between the two of them is ovulation which is a function of upsurge of estrogen. Menstruation which is really the shedding of endometrium in preparation for the proliferation is embedded in the proliferative phase. This understanding has created a template for human physiologist and obstetricians for the study of fertility, infertility and contraception in human. Furthermore, it has helped in projecting non-invasive methods for population control. However, this hormonal interplay also affects other systems such as the respiratory and cardiovascular systems. For instance studies have revealed that ventilator response is increased during the progesterone dominated luteal phase at rest (Schoene et al., 1981; White et al., 1983) and at exercise (Williams and Krahenbuhl, 1997). Progesterone has also been reported to have a role in relaxation of bronchial smooth muscle which reduces the contractile response of the respiratory muscles (Mannan et al., 2007). The estrogen dominated follicular phase has been shown to be associated with increased expiratory resistance (Anderson and Babcock, 2008). For the cardiovascular system, the mid luteal phase has been shown to be associated with marked decrease in total peripheral resistance and a significant decrease in mean arterial blood pressure (Hassan et al., 1990). Systemic blood pressure increases relatively at the onset of the menstrual phase when compared to the other phases of the menstrual cycle (Dunne et al., 1991). However, conflicting data reported the causative changes in blood pressure during the normal menstrual cycle (Greenberg et al., 1985). This study is therefore aimed at comparing the peak expiratory flow rate (PEFR) and the pulse pressure during the luteal and menstrual phases of the menstrual cycle.

\section{MATERIALS AND METHOD}

The cross sectional study was done among apparently healthy girls of the St Philomena School of Midwifery after obtaining clearance from the ethics and collaboration committee of the institution. A total of 60 volunteers comprising 30 subjects in the late luteal phase of the menstrual cycle and 30 subjects in the menstruation phase of the menstrual cycle participated in the study. Both groups had subjects with age, height and weight between $20-25$ years, $155-165 \mathrm{~m}$ and $55 \mathrm{~kg}-65 \mathrm{~kg}$ respectively. An informed consent was obtained from each subject. However, any subject with any of the exclusion criteria (Omorogiuwa and Iyawe, 2009) for Peak Expiratory Flow Rate was disqualified from the study. Prior to peak expiratory flow measurement, subjects were asked to empty their bladder and wear loosed clothing. Measurement of peak expiratory flow rate 
(PEFR) was initiated with the subject sitting in an upright position. Each subject was instructed on the proper technique for measuring PEFR using Wright's peak flow meter, which had been calibrated. For each PEFR measurement, the subject was asked to breathe in as deeply as possible. Place the mouth piece in the mouth; lightly bite with the teeth closing the lips on it and with the hand press of the nostril to avoid escape of air. Blow air into the instrument mouth piece as hard and fast as possible. The above procedure was done three times consecutively records were taken and the highest test flow rate was recorded on liters per minutes and used for analysis.

Following a rest period of about 30 minutes, the systolic and diastolic pressures were measured in each subject in the sitting position on the brachial artery using auscultatory method. Diastolic pressure was determined as the disappearance of the Korotkoff's sound. The pulse pressure was calculated from the difference of the measured systolic and diastolic pressures. Expressed mathematically as; $\mathrm{P}_{\text {Pressure }}=\mathrm{P}_{\text {systolic }}-\mathrm{P}_{\text {diastolic }}$. Height was measured in meters using a standard Stadiometer, Weight was measured in kilogram using the Bathroom Hana

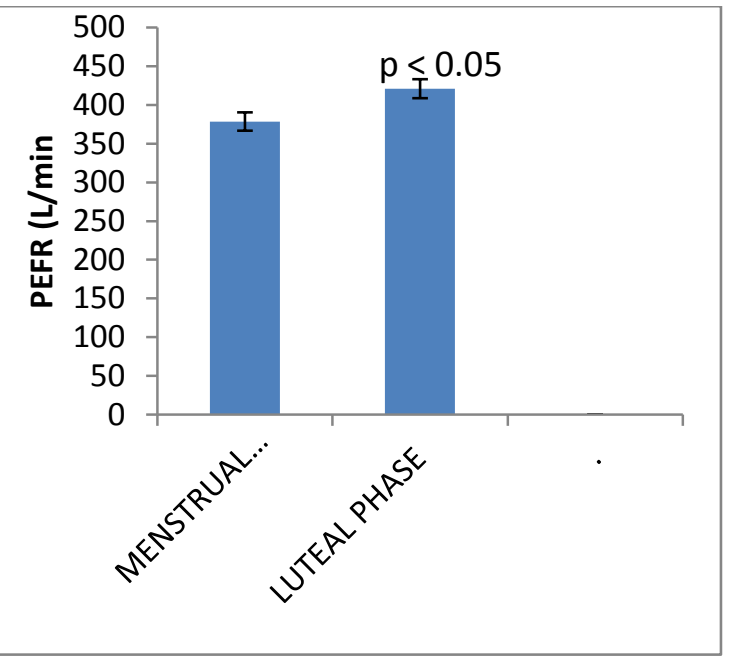

Fig 1: Peak Expiratory Flow Rate (PEFR) in the Menstrual and luteal phases of the menstrual cyclep $<0.05$ indicates a statistical significant difference

The menstrual cycle is a four phased series of uteroovarian and hormonal events that occur in fertile women and other primates for the purpose of fertilization and implantation (Omorogiuwa and Egbeluya, 2014). The phases are menstruation, proliferative, ovulation and secretory. The hormonal components have some cardio-respiratory influence on the female during the menstrual cycle. The study revealed a strengthening of the respiratory function
Scale. BMI was calculated as weight in kilograms divided by height in meters squared $\left(\mathrm{BMI}=\mathrm{kg} / \mathrm{m}^{2}\right)$.

Statistical Analysis: Results were presented in Bar charts using Microsoft excel 2010. Appropriate statistical comparisons were made using the student's t-test and $\mathrm{p}<0.05$ was considered significant.

\section{RESULTS AND DISCUSSION}

A total of sixty subjects volunteered for the study with thirty subjects in the luteal phase of the menstrual cycle and thirty in the menstruation phase. However, three subjects in the luteal phase of the menstrual cycle declined as per study protocol, while five subjects in the menstruation phase declined as per study protocol. Thus, 27 and 35 subjects in the luteal phase and menstruation phase respectively took part in the study with $10 \%$ attrition for the luteal phase group and $16.7 \%$ attrition for the subjects in the menstruation phase. The ages of the subjects in the menstrual and luteal phases of the menstrual cycle were $20.80 \pm 0.46$ and $20.40 \pm 0.37$ respectively. There was no statistical significant difference $p>0.05$ between the two age groups.

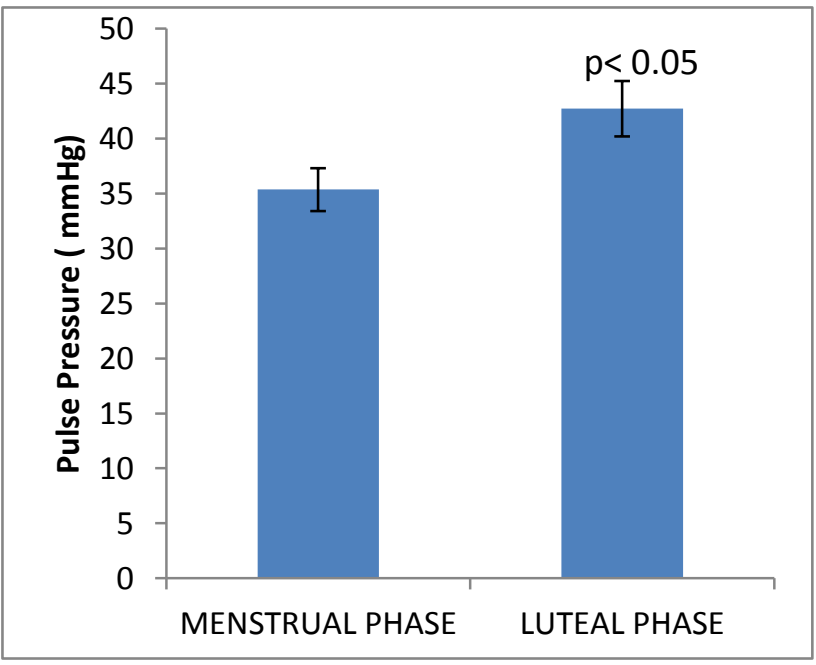

Fig 2: Pulse pressure in the menstruation and luteal phases of the menstrual cyclep $<0.05$ indicates a statistical significant difference

i.e. increased peak expiratory flow rate during the luteal phase compared to the menstruation phase of the menstrual cycle (figure 1). The maximum inflation and deflation is an important physiological stimulus for the release of surfactant and prostaglandins. These substances increases alveolar spaces and decrease bronchial smooth muscle tone activity with an overall increase in lung compliance (Hildebran et al., 1981; Smith, 1976). In this study, 
we recorded a relative increase in the peak expiratory flow rate during the luteal phase compared to the value in the menstruation phase of the menstrual cycle (figure 1). The effect of progesterone on pulmonary function during the luteal phase may be the explanation for the increase observed in this study. The stimulatory effect of progesterone on the increased mRNA content of progesterone receptor at the hypothalamus during the luteal phase results to hyperventilation (Ganong, 2006) and an improved pulmonary function (Pai et al., 2004, Kaygisiz et al., 2003, Okagaki and Richart, 1970). Although prostaglandin release is higher during menstruation, its positive effect on respiration function was surpassed by the progesterone dominated luteal phase of the menstrual cycle. Elevated progesterone level and core temperature during the luteal phase of the menstrual cycle have been implicated in the increase in ventilation (White et al., 1996). Thus one can assert that bronchial smooth muscle relaxation and the corresponding lung compliance is optimal during the luteal phase of the menstrual cycle. This finding can be of advantage to girls in the reproductive age group who probably will be performing better in athletic sports competition during the luteal phase. Furthermore, the increased peak expiratory flow rate observed in the luteal phase of this study may exempt asthmatic from attacks during this period. This has to be borne in mind during management and planning of therapeutic regimens in female patients with asthma (Pai et al., 2004). This study revealed an increase in pulse pressure in the luteal phase of the menstrual cycle compared to the menstruation phase (figure 2 ).
This relative increase in pulse pressure is traceable to the decreased diastolic blood pressure (figure 3). On the contrary, the relative decrease in pulse pressure during menstruation when compared to the luteal phase of the menstrual cycle is a function of the increased diastolic blood pressure (figure 3). This corroborates the previous studies which reported a marked decrease in total peripheral resistance and mean arterial blood pressure in the mid luteal phase (Hassan et al., 1990). Furthermore, Dunne et al (1991) reported a high blood pressure at the onset of menstrual phase compared to other phases of the menstrual cycle. The pulse rate of the subjects during the luteal phase was higher than the value during the menstruation phase. This increase however was only empirical as there was no statistical difference between the phases of the menstrual cycle studied (figure 4). Anthropometric parameters such as age, sex, weight and height have also been documented to affect pulmonary function (Alakija et al., 1990; Cotes et al.,1966; Jain et al.,1983) The difference in body mass indices of both groups studied (figure 5) was not statistically significant $(\mathrm{p}>0.05)$. Consequently, the progesterone dominance in the luteal phase is accountable for the relative enrichment in pulmonary function in this phase compared to the menstrual phase.

The findings from this study thus suggest that there is an improved cardiopulmonary function during the luteal phase of the menstrual cycle compared to the menstruation phase and this should be taken into consideration by health care givers.

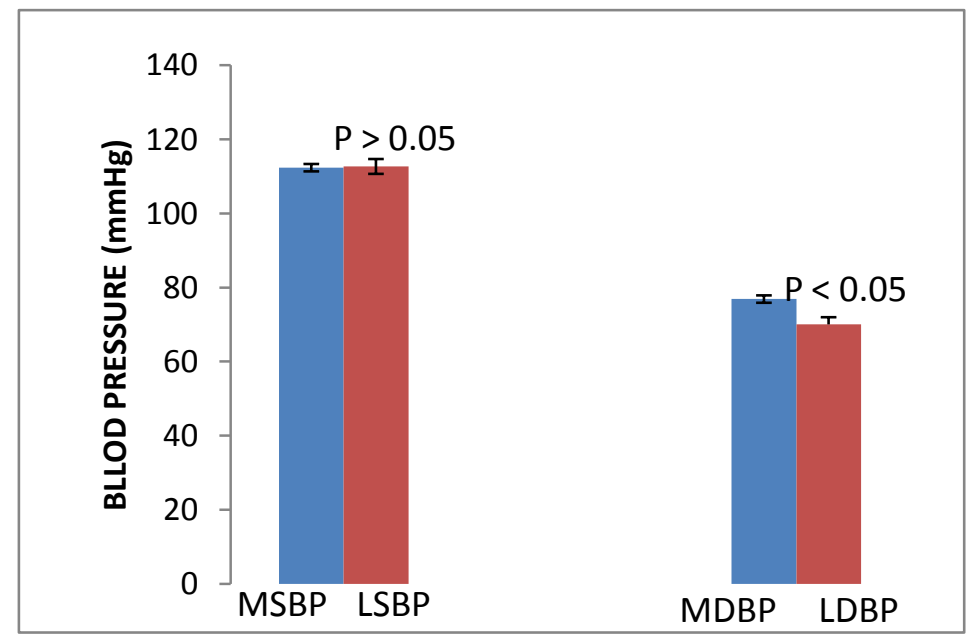

Fig 3: Menstrual Systolic Blood Pressure (MSBP), Luteal Systolic Blood Pressure (LSBP), Menstrual Diastolic Blood Pressure (MDBP), Luteal Diastolic Blood Pressure (MDBP. $\mathrm{p}<0.05$ indicates a statistical Significance, while $\mathrm{p}>0.05$ indicates no statistical difference

*I OMOROGIUWA, A; OJIEMUDIA, $M$ 


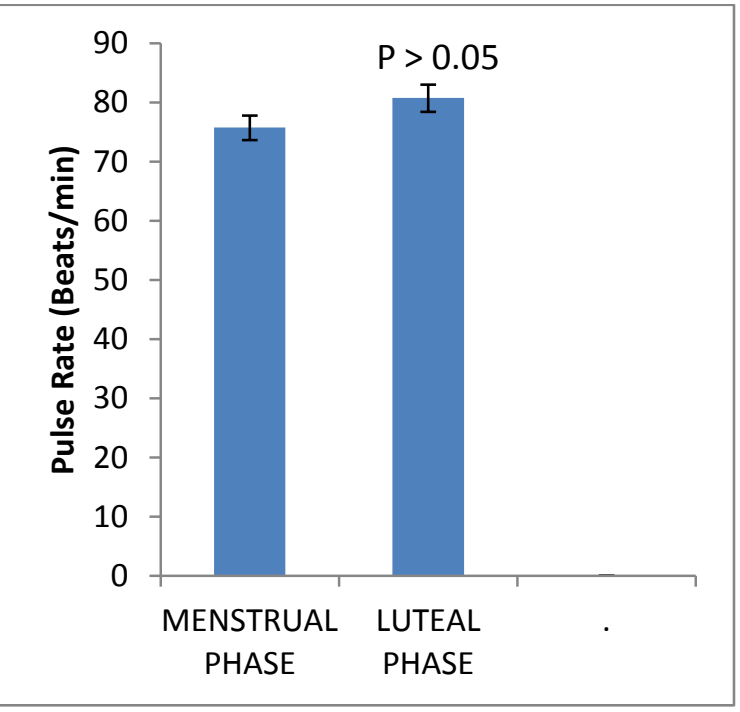

Fig 4: Pulse rates during the luteal and menstrual phases of the menstrual cycle. $p>0.05$ indicates no statistical significant difference

\section{REFERENCES}

Alakija W, Iyawe VI, Jarikre LN, Chiwuzie JC. (1990). Venilatory function of workers at Okpella Cement factory in Nigeria. W. Afr. J. Med. 9(3):187-192.

Anderson AJ, Babcock MA. (2008). Effects of the menstrual cycle on expiratory resistance during whole body exercise in females. Journal of Sports science and Medicine.7: 475-479.

Cotes JE, Russiter CE, Higgins ITT, Gilson JC. (1966). Average normal values for the forced expiratory volume in white Caucasian males. $\mathrm{Br}$ Med J. 1(5494):1016-1019

Dunne FP, Barry DG, Ferriss JB, Grealy G, Murphy D (1991). Changes in blood pressure during normal menstrual cycle. Clin Sci. 81: 515-518.

Greenberg G, Imeson JD, Thompson SG, Meade TE. (1985).Blood pressure and the menstrual cycle. Br. J. Obstet. Gynecol. 92(10): 1010-1014.

Golparvar M, Ahmadi F, Saghaei M. (2005). Effects of progesterone on the ventilatory performance in adult patients during partial support mechanical ventilation. Archives of Inanian Medicine 8(1): 27-31.

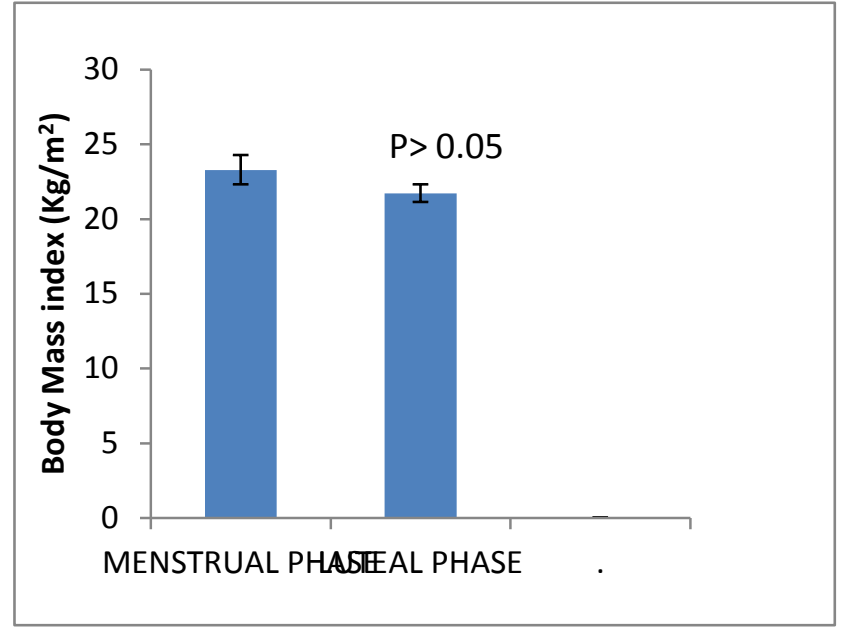

Fig 5: Body Mass Index (BMI) of subjects in the menstrual phase and the luteal phase of the menstrual cycle $p>0.05$ indicates no statistical significant difference

Hassan AA, Carter G and Tooke JE. (1990). Postural vasoconstriction in women during normal menstrual cycle. Clin. Sci. 78: 39-47.

Hildebran JN, Goerke J, Clements JA. (1981). Surfactant release in excised rat lung is stimulated by air inflation. J Appl Physiol. 51(4):905-10.

Jain SK, Kumar R, Sharma DA. (1983) Factors influencing peak expiratory flow rate (PEFR) in normal subjects. Lung India. 1(3):92-97.

Kaygisiz Z, Erkasap N, Soydan M. (2003). Cardiorespiratory responses to submaximal incremental exercise are not affected by night's sleep deprivation during the follicular and luteal phases of the menstrual cycle. Indian J. Physiol. and Pharmacol. 47 (3): 279-287.

Mannan SR, Begum N, Begum S, Ferdousi S, Ali T (2007).Relationship of forced vital capacity (FVC), Forced expiratory volume in first second (FEV1) and FEV1/FVC\% with plasma progesterone level during different phases of normal menstrual cycle. J. Bangladesh Soc. Physiol. 2:7-12.

Okagaki T, Richart RM. (1970).Oxygen consumption of human endometrium during the menstrual cycle. Fertility Sterility. 21(8): 595-598. 
Omorogiuwa A, Iyawe VI. (2009). Peak expiratory flow rate in the three trimesters of pregnancy. Journal of Medicine and Biomedical Research. $8(2): 19-23$

Omorogiuwa A, Egbeluya EE. (2014). A comparative study of the hematological values in the Ovulation and Luteal phases of the menstrual cycle. Int.J.Biol.Chem.Sci. 2014; 8(4): 1853-1858

Pai SR, Prajna P, D’Souza UJA (2004). A Correlative study on blood pressure and lung function profiles during different phases of menstrual cycle among Indian population.Thai. J. of Physio. Sci. 17(2): 30-34.

Schoene JB, Robertson HT, Pierson D, Peterson AP (1981). Respiratory drive and exercise in menstrual cycle of athletic and nonathletic women. J. Appl. Physiol. 50: 1300-1305.
Smith, AP (1976). Prostaglandins and respiratory system prostaglandins; Physiological, Pharmacological and Pathological Aspects. Edited by SMM. Karim. p83-102.

Takano N. (1984). Reflex hypoxic drive to respiration during the menstrual cycle. Respir. Physiol. 56: 229-235.

Williams TJ, Krahenbuhl GS (1997). Menstrual cycle phase and running economy. Med. Sci. Sports Exercise. 29: 1609-1618.

White DP, Douglas NJ, Pickett CK, Weil JV, Zwilliche W. (1983). Sexual influence on the control of breathing. J. Appl. Physiol. Respir. Environ. Exercise Physiol.54: 874-879.

White MD, Cabanac M. (1996). Exercise hyperpnoea and hypothermia in humans. J Appl Physiol. 81(3):1249-54. 